\title{
Efficient algorithm for solving semi-Infinite programming problems and their applications to nonuniform filter bank designs
}

Ho, Charlotte; Ling, Bingo Wing-Kuen; Liu, Yanqun; Tam, Peter; Teo, Kok Lay

https://researchrepository.rmit.edu.au/esploro/outputs/9921859584001341/filesAndLinks?institution=61RMIT_INST\&index=null

Ho, C., Ling, B. W.-K., Liu, Y., Tam, P., \& Teo, K. L. (2006). Efficient algorithm for solving semi-Infinite programming problems and their applications to nonuniform filter bank designs. IEEE Transactions on Signal Processing, 54(11), 4223-4232. https://doi.org/10.1109/TSP.2006.880314

Published Version: https://doi.org/10.1109/TSP.2006.880314

Repository homepage: https://researchrepository.rmit.edu.au (c) 2006 IEEE. Personal use of this material is permitted. However, permission to reprint/republish this material for advertising or promotional purposes or for creating new collective works for resale or redistribution to servers or lists, or to reuse any copyrighted component of this work in other works must be obtained from the IEEE.

Downloaded On 2023/04/27 01:22:45 +1000 


\title{
Efficient Algorithm for Solving Semi-Infinite Programming Problems and Their Applications to Nonuniform Filter Bank Designs
}

\author{
Charlotte Yuk-Fan Ho, Bingo Wing-Kuen Ling, Yan-Qun Liu, Peter Kwong-Shun Tam, Member, IEEE, and \\ Kok-Lay Teo, Senior Member, IEEE
}

\begin{abstract}
An efficient algorithm for solving semi-infinite programming problems is proposed in this paper. The index set is constructed by adding only one of the most violated points in a refined set of grid points. By applying this algorithm for solving the optimum nonuniform symmetric/antisymmetric linear phase finite-impulse-response (FIR) filter bank design problems, the time required to obtain a globally optimal solution is much reduced compared with that of the previous proposed algorithm.
\end{abstract}

Index Terms-Dual parameterization, nonuniform filter banks, semi-infinite programming.

\section{INTRODUCTION}

$\mathbf{M}$ ANY engineering problems, such as, parametric estimation problems [1], identification problems [2], array pattern synthesis problems [3], window, filter, filter bank, and wavelet kernel design problems [4]-[19], as well as optimal control problems [20]-[24], can be formulated as optimization problems subject to functional inequality constraints. Since continuous functions consist of infinite number of discrete points, these infinite constraint optimization problems cannot be solved via some simple methods.

In order to solve these problems, numerically efficient simplex extension algorithms were employed in [6] and [16];

Manuscript received April 19, 2005; revised November 11, 2005. The associate editor coordinating the review of this manuscript and approving it for publication was Dr. Henrique S. Malvar. The work obtained in this paper was supported by a research grant (project number G-YD26) from The Hong Kong Polytechnic University, the Centre for Multimedia Signal Processing, The Hong Kong Polytechnic University, the CRGC grant (Project Number PolyU 5105\01E) from the Research Grants Council of Hong Kong, as well as a research grant from the Australian Research Council.

C. Y.-F. Ho is with the Department of Electronic Engineering, Queen Mary, University of London, London E1 4NS, U.K. (e-mail: charlotte.ho@elec.qmul.ac.uk).

B. W.-K. Ling is with the Department of Electronic Engineering, Division of Engineering, King's College London, London WC2R 2LS, U.K. (e-mail: wing-kuen.ling@kcl.ac.uk).

Y.-Q. Liu is with the Department of Mathematics and Statistics, Royal Melbourne Institute of Technology, Melbourne VIC 3001, Australia (e-mail: yanqun.liu@rmit.edu.au).

P. K.-S. Tam is with the Department of Electronic and Information Engineering, The Hong Kong Polytechnic University, Kowloon, Hong Kong, China (e-mail: enptam@polyu.edu.hk).

K.-L. Teo is with the Department of Mathematics and Statistics, Curtin University of Technology, Perth, CRICOS Provider Code 00301J, Australia (e-mail: K.L.Teo@curtin.edu.au).

Digital Object Identifier 10.1109/TSP.2006.880314 conventional dual parameterization approaches were employed in [5], [12], [17], [18] and [27]; an extended active set strategy was employed in [4]; an extended version of Remez algorithm was employed in [7] to generate a feasible starting condition for the dual method; discretization methods were employed in [9], [15], and [16]; outer-approximation cutting plane algorithms were employed in [10], [15], and [30]; penalty function approaches were employed in [11] and [26]; and constraint transcription approaches were employed in [13] and [25]. Among them, the constraint transcription methods, the penalty function approaches, the discretization methods, the cutting plane methods and the conventional dual parameterization methods are the most common methods employed for solving engineering problems.

However, for the constraint transcription method [25], it suffers from convergence problems. For the penalty function approach [11], it suffers from disadvantages of computing numerical integration. For the discretization method [9], it does not guarantee that the solution obtained would satisfy the corresponding continuous constraints. For the cutting plane method [30], the corresponding subset of the index set may consist of infinite number of points and only approximated solution is obtained. On the other hand, the dual parameterization method is capable of finding the exact solution because a global solution of the parameterized dual problems provides a solution to the primal problems.

For the conventional dual parameterization method, it is to parameterize the measure $\boldsymbol{\mu}$ in the dual problems so that it transforms the semi-infinite programming problems into equivalent finite dimensional nonlinear programming problems via sequences of regular convex programs. These sequences of convex programs are obtained by discretization. In the $k$ th iteration, the problems are solved by replacing the index set $\Delta$ with a finite subset $\Delta_{k}$. The finite subset $\Delta_{k+1}$ of the index set is constructed from $\Delta_{k}$ by adding all violated index points of a refined set of grid points to $\Delta_{k}$ while dropping all unnecessary points from $\Delta_{k}$. However, as the number of all violated index points in a continuous set could be very large or even infinite, so the shortcoming of this scheme is that the number of points in $\Delta_{k}$ may be unbounded as $k$ increases. For example, the number of points that violates $-x^{2}>-1$ is infinity because there are infinite number of points in the set $x \in(-1,1)$. Even if the number of points in $\Delta_{k}$ is finite, the computational complexity 
of employing this approach for solving the problem may be very huge because the number of points in $\Delta_{k}$ may be very large. In this paper, this conventional dual parameterization method is improved as follows. We construct $\Delta_{k+1}$ from $\Delta_{k}$ by adding to $\Delta_{k}$ only one of the most violated points in a refined set of grid points. Hence, the number of points in $\Delta_{k}$ is bounded. For the same example, the most violated point is at $x=0$ and we only add this point in $\Delta_{k}$.

It is shown in this paper that for each $k$, the number of points in $\Delta_{k}$ is limited by the number of filter coefficients required to be designed plus two. The computational complexity of the dual parameterization algorithm does not only depend on the filter lengths, but also on the number of points in the refined sets of grid points. Hence, the computational complexity of this algorithm is greatly reduced compared to the conventional one [27]. It is worth noting that both the order of the optimization problem and the number of points in the refined sets of grid points for the nonuniform filter bank design problems are high. This is because more than one filter is required to be designed and the orders of the polynomials for the amplitude and aliasing distortions are much higher than that for the specifications on the passband and stopband of the filters. Hence, the complexity issue is particularly important for the nonuniform filter bank design problems.

The rest of this paper is organized as follows. In Section II, we summarize the formulation of the optimum nonuniform symmetric/antisymmetric linear phase FIR filter bank design problem as a quadratic semi-infinite programming problem [18]. In Section III, an improved dual parameterization algorithm for solving the semi-infinite programming problem is proposed. In Section IV, a numerical experiment for this nonuniform filter bank design problem is presented. Finally, Section V concludes the paper.

\section{PROBLEM FORMULATION}

In this paper, either symmetric or antisymmetric FIR filters are designed, so all the filters are linear phase. The formulation of this optimum nonuniform symmetric/antisymmetric linear phase FIR filter bank design problem is similar to that of the nonuniform transmultiplexer design problem [18], so only the summary of the formulation is stated in this paper. Interested readers are recommended to study [18].

Let the coefficient vectors of the analysis and synthesis filters be, respectively, $\mathbf{x}$ and $\mathbf{y}$. By arbitrarily choosing a set of synthesis filters, $\mathbf{y}$ is fixed. Define the cost function of an optimization problem as the sum of the ripple energy for all the individual filters in the passband and stopband subject to the specifications on the passband and stopband ripple magnitudes, as well as on the amplitude and aliasing distortions. Using similar approach in [18], the nonuniform filter bank design problem can be formulated as the following semi-infinite programming problem:

\section{Problem (P)}

$$
\begin{array}{cc}
\min _{\mathbf{x}} & J(\mathbf{x})=\frac{1}{2} \mathbf{x}^{T} \mathbf{Q} \mathbf{x}+\mathbf{b}^{T} \mathbf{x}+p \\
\text { subject to } & \mathbf{g}(\mathbf{x}, \omega)=\mathbf{A}(\omega) \mathbf{x}-\mathbf{c}(\omega) \leq \mathbf{0}, \quad \text { for } \omega \in \Delta
\end{array}
$$

where $J(\mathbf{x})$ is the cost function, $\mathbf{g}(\mathbf{x}, \omega) \leq \mathbf{0}$ is the continuous constraint function, $\mathbf{Q}$ is a positive definite matrix, $\mathbf{b}$ is a vector, $p$ is a positive scalar, $\mathbf{A}(\omega)$ and $\mathbf{c}(\omega)$ are continuously differentiable functions with respect to some frequency band $\Delta$.

Since amplitude and aliasing distortions are complex continuous functions, constraints that take absolute values on these functions are equivalent to quadratic constraints, which cause the problem to be very difficult to solve. One common way to tackle this difficulty is via a discretization approach. If the discretization approach is employed, then the constraints become convex quadratic constraints which will lead to a quadratic programming problem. However, the number of grid points required is very large. As a result, the computational complexity is very high. Hence, in this formulation, we make an approximation that the constraints on taking absolute values of these complex functions are almost (but not) equivalent to constraints on both the real and imaginary parts of these functions. The approximation is valid if the constraint functions are small. By making such approximation, the quadratic constraints can be approximated as linear constraints and the problem is much simplified and become easier to solve.

Problem $\mathbf{P}$ can be efficiently solved using the improved dual parameterization method and discussed in the following section in detail. The problem is convex, so any local solution is a global solution and the solution obtained is independent of the initial values of $\mathbf{x}$ and $\mathbf{y}$ we select. Once we obtain the set of analysis filters, we use the same method to compute the synthesis filters, and iterate the above procedures. Since the feasible set of each iteration is convex, this iteration process is actually a projection on a convex set. Hence, the convergence of the iteration process is guaranteed if a solution exists for each iteration. Eventually, a set of analysis and synthesis filters that globally minimizes the cost function and satisfies the continuous constraints is obtained.

\section{IMPROVED DUAL-PARAMETERIZATION ALGORITHM}

As discussed in Section I, the conventional dual parameterization method is to parameterize the measure in the dual problems so that it transforms the semi-infinite programming problems into equivalent finite dimensional nonlinear programming problems via sequences of regular convex programs. The basic working principle of the conventional dual parameterization method can be summarized as follows: Since the constraint functions are continuous with respect to their index parameters and the index set is compact Hausdorff, the constraint functions can be redefined as an operator whose range is the Banach space consisting of continuous functions defined on the index set and equipped with the uniform norm. The order in the range space is given by a cone consisting of all nonnegative functions on the index set. The assumption of the conventional dual parameterization method is the existence of a solution that strictly satisfies the continuous constraints. This condition is also known as the Slater's condition. Once the Slater's condition is satisfied, the Karush-Kuhn-Tucker (KKT) conditions would be satisfied, which guarantees a necessary optimality condition for such a cone-constrained nonlinear programming problem, where the Lagrange multiplier is defined as a regular Borel measure on the index set. As a result, the set of multipliers satisfying the KKT condition necessarily includes a measure with finite support unless it is empty. Hence, any constraint qualification ensures the existence of such a discrete measure, which is also called 
the Haar measure. On the other hand, strong duality holds for convex programming under Slater's constraint qualification. Hence, the corresponding dual problem for semi-infinite programming can then be formulated in the space of finite signed regular Borel measures on the index set. The local KKT theory and the global duality theory are naturally related through the fact that the set of multipliers satisfying the KKT condition coincides with the set of solutions to the dual problem, which leads to the consequence that the set of dual solutions always includes a measure with finite support under the Slater condition. Hence, the conventional dual parameterization method is guaranteed to obtain a globally optimal solution that satisfies the continuous constraint if a solution exists.

In order to reduce the computational complexity of the conventional dual parameterization method, we need to define the dual problem, the Slater condition and the KKT condition as follows.

The Dorn's dual of problem $\mathbf{P}$ can be formulated as shown in the equation at the bottom of the page, where $\Lambda^{+}(\Delta)$ is the set of nonnegative bounded regular Borel measures on $\Delta$.

As discussed in the above, in order to solve the problem sequence, we need an assumption of the satisfaction of the Slater condition, and it is stated as follows.

Assumption 1 Slater Condition: $\exists \mathrm{x}_{0} \in \Re^{S}$ such that $\mathbf{g}\left(\mathbf{x}_{0}, \omega\right)<\mathbf{0}, \forall \omega \in \Delta$, where $S$ is the dimension of the vector $\mathbf{x}_{0}$.

For the nonuniform filter bank design problem, this assumption can be interpreted as follows: There exists a set of filter coefficients such that the maximum values of the amplitude and aliasing distortions, as well as the passband and stopband ripple magnitudes of the filters, are strictly lower than that of the required specifications. If the set of decimation integer is compatible, then this assumption can be easily achieved by using a set of filters with suitably long filter lengths.

As discussed in the above, the dual parameterization technique is based on the dual parameterization theory, in which the dual parameterization theory is based on the following results.

Lemma 1 KKT Condition: Assume that the Slater constraint qualification is satisfied. The minimum of problem $\mathbf{P}$ is achieved at $\mathbf{x}^{*}$ if and only if $\mathbf{x}^{*}$ is feasible and there exists a $\nu^{*} \in \Lambda(\Delta)$ such that

$$
\begin{array}{r}
\mathbf{Q x}^{*}+\mathbf{b}+\int_{\Delta}(\mathbf{A}(\omega))^{T} d \boldsymbol{\nu}^{*}(\omega)=\mathbf{0} \\
\int_{\Delta}\left(\mathbf{A}(\omega) \mathbf{x}^{*}-\mathbf{c}(\omega)\right)^{T} d \boldsymbol{\nu}^{*}(\omega)=0
\end{array}
$$

and

$$
\nu^{*} \geq 0
$$

where $\Lambda(\Delta)$ denotes the space of all signed finite regular Borel measure.

This lemma states the necessary and sufficient condition relating the minimum of problem $\mathbf{P}$ and the existence of $\boldsymbol{\nu}^{*}$ in the space of all signed finite regular Borel measure. This result will be used in Lemmas 3 and 4 later. Since this result is well known in the optimization community [28], we just state it and omit the proof. Interested readers are recommended to study [28].

Lemma 2 Carathéodory Theorem: Let $U$ be a subset of $\Re^{S}$. If $\mathbf{x} \in$ cone $U \equiv \bigcup_{\boldsymbol{\alpha}>\mathbf{0}} \boldsymbol{\alpha} c o U$, that is, $\mathbf{x}$ is a nonnegative linear combination of points in $U$, then there exists $S$ numbers of $\alpha_{i} \geq$ 0 such that $\mathbf{x}=\sum_{i=1}^{S} \alpha_{i} \mathbf{x}_{i}^{c}$ for some $\mathbf{x}_{i}^{c} \in U, i=1,2, \ldots, S$. In order words, if $\mathbf{x} \in$ cone $U$, then $\mathbf{x}$ can be represented as a nonnegative linear combination of at most $S$ points of $U$.

This lemma states that the measure $\boldsymbol{\nu}^{*}$ has a finite support of no more than $S$ points. This lemma is also required for the proof of Lemma 4 which is stated later. Since this result is also well known in the optimization community [28], we just state it and omit the proof. Again, interested readers are recommended to study [28].

Lemma 3: Assume that the Slater constraint qualification is satisfied and the minimum of problem $\mathbf{P}$ is achieved at $\mathbf{x}^{*}$. Then $\boldsymbol{\nu}^{*}$ is a multiplier satisfying the KKT condition if and only if $\left(\mathbf{x}^{*}, \nu^{*}\right)$ is a solution to the dual problem DP.

This lemma states the relationship between the satisfaction of KKT condition stated in Lemma 1 and the solution to the dual problem DP. This lemma is also required for the proof of Lemma 4 which is stated later. Since the proof is shown in detail in [27], we omit the proof in this paper. Interested readers are recommended to study [27].

Lemma 4: Assume that the Slater constraint qualification is satisfied and the minimum of problem $\mathbf{P}$ is achieved at $\mathbf{x}^{*}$. Then the solution set of the dual problem DP contains a solution pair $\left(\mathrm{x}^{*}, \boldsymbol{\nu}^{*}\right)$ of which the measure $\boldsymbol{\nu}^{*}$ has a finite support of no more than $S$ points.

Based on the KKT condition stated in Lemma 1, the Carathéodory Theorem stated in Lemma 2 and the relationship between the satisfaction of KKT condition and the solution to the dual problem DP stated in Lemma 3, the proof of Lemma 4 can be followed easily. Since the detail proof is also shown in [27], we omit the proof in this paper. Interested readers are recommended to study [27].

Lemma 4 is the foundation of the dual parameterization method. The importance of Lemma 4 is that it allows us to reduce problem DP to a finite dimensional problem. In order to solve the primal problem $\mathbf{P}$, we only need to find a solution pair $\left(\mathbf{x}^{*}, \boldsymbol{\nu}^{*}\right)$ of problem DP. From Lemma 4 , we can restrict our search for $\boldsymbol{\nu}^{*}$ to those nonnegative measures having a finite support of no more than $S$ supporting points. Such a measure $\boldsymbol{\nu}$ is characterized by its $k$ supporting points

$$
\begin{array}{cc}
\underset{\substack{\mathbf{x}, \boldsymbol{\nu} \\
\text { Problem }}}{\operatorname{DP})} & \frac{1}{2} \mathbf{x}^{T} \mathbf{Q x}+\int_{\Delta}(\mathbf{c}(\omega))^{T} d \boldsymbol{\nu}(\omega) \\
\text { subject to } & \mathbf{Q x}+\mathbf{b}+\int_{\Delta}(\mathbf{A}(\omega))^{T} d \boldsymbol{\nu}(\omega)=\mathbf{0}, \quad \boldsymbol{\nu} \in \Lambda^{+}(\Delta)
\end{array}
$$


$\omega_{i} \in \Delta$ for $i=1,2, \ldots, k$, and the corresponding measures $\boldsymbol{\mu}_{i}=\boldsymbol{\nu}\left(\left\{\omega_{i}\right\}\right)>\mathbf{0}$ for $i=1,2, \ldots, k$ at each point. If we restrict the measure $\nu$ in problem DP to those of finite support of no more than $k$ supporting points which are collectively denoted by $Z$, then problem $\mathbf{D P}$ is reduced to the following problem:

$$
\begin{aligned}
& \text { Problem }\left(\mathbf{P D P}_{k}\right) \\
& \min _{\mathbf{x}, \boldsymbol{\mu}, \boldsymbol{\tau}} \frac{1}{2} \mathbf{x}^{T} \mathbf{Q} \mathbf{x}+\sum_{i=1}^{k}\left(\mathbf{c}\left(\omega_{i}\right)\right)^{T} \boldsymbol{\mu}_{i} \\
& \text { subject to } \mathbf{Q x}+\mathbf{b}+\sum_{i=1}^{k}\left(\mathbf{A}\left(\omega_{i}\right)\right)^{T} \boldsymbol{\mu}_{i}=\mathbf{0} \\
& \boldsymbol{\mu}_{i} \geq \mathbf{0}, \quad \text { for } i=1,2, \ldots, k \\
& \omega_{i} \in \Delta, \quad \text { for } i=1,2, \ldots, k
\end{aligned}
$$

Problem $\mathbf{P D P}_{k}$ is called the parameterized dual of problem $\mathbf{P}$ with parameterization number $k$. From the above discussions, we see that once a global solution $\left(\mathbf{x}^{*}, \boldsymbol{\mu}^{*}, \boldsymbol{\tau}^{*}\right)$ of problem $\mathbf{P D P}_{k}$ is obtained, then $\mathbf{x}^{*}$ must be the solution of problem $\mathbf{P}$ if $k$ is suitably large. Here, suitably large means that $k$ is no less than an integer $k^{*}$, the minimum parameterization number, which is no more than $S$, but not known exactly before solving the problem. Thus, in order to solve problem $\mathbf{P}$, we only need to deal with problem $\mathbf{P D P}_{k}$.

For any finite index set $Z=\left\{\omega_{1}, \omega_{2}, \ldots, \omega_{k}\right\} \subset \Delta$, we define the following problem:

$$
\begin{gathered}
\text { Problem } \underset{\mathbf{x}, \boldsymbol{\mu}}{\mathbf{P D P}}(Z))_{\frac{1}{2} \mathbf{x}^{T} \mathbf{Q} \mathbf{x}+\sum_{j=1}^{k}\left(\mathbf{c}\left(\omega_{j}\right)\right)^{T} \boldsymbol{\mu}_{j}} \\
\text { subject to } \\
\mathbf{Q} \mathbf{x}+\mathbf{b}+\sum_{j=1}^{k}\left(\mathbf{A}\left(\omega_{j}\right)\right)^{T} \boldsymbol{\mu}_{j}=\mathbf{0} \\
\boldsymbol{\mu}_{j} \geq \mathbf{0}, \quad \text { for } j=1,2, \ldots, k
\end{gathered}
$$

where $\boldsymbol{\mu}$ is defined as in problem $\mathbf{P D P} \mathbf{P}_{k}$.

Hence, problem $\mathbf{P}$ can be solved efficiently by the following improved dual parameterization algorithm. The algorithm combines an adaptive scheme for an approximated solution and a local search procedure.

For each $i \geq 1$, let $\Delta_{i}$ be a given subset of $\Delta$ satisfying

$$
d\left(\Delta_{i}, \Delta\right) \equiv \max _{\varpi \in \Delta} \min _{\omega \in \Delta_{i}}|\varpi-\omega| \rightarrow 0 .
$$

For any $\omega \in \Delta$ and $\mathbf{x} \in \Re^{S}$, we define $g_{\max }(\mathbf{x}, \omega) \equiv$ $\max _{1 \leq j \leq m} g_{j}(\mathbf{x}, \omega)$, where $g_{j}(\mathbf{x}, \omega)$ is the $j$ th entry of the vector $\mathbf{g}(\mathbf{x}, \omega)$ and $m$ is the number of rows of $\mathbf{g}(\mathbf{x}, \omega)$.

Algorithm 1:

Step 1) Choose an arbitrary filter coefficient vector $\mathbf{x}^{0} \in \Re^{S}$, a small number $\epsilon>0$, a large integer $N$, and a sequence of finite parameterization sets $\Delta_{i}=\left\{\omega_{j}^{i}\right.$ : $\left.j=1,2, \ldots, k_{i}\right\}$, for $i=1,2, \cdots$, satisfying (2).

Step 2) Let $E_{0}=\oslash$, where $\oslash$ denotes the empty set. Set $i=0$.

Step 3) Set $i=i+1$. Find $\varpi_{i} \in \Delta_{i}$ such that $g_{\max }\left(\mathbf{x}^{i-1}, \varpi_{i}\right)=\max _{\omega \in \Delta_{i}} g_{\max }\left(\mathbf{x}^{i-1}, \omega\right)$.

If $g_{\max }\left(\mathrm{x}^{i-1}, \varpi_{i}\right)<\epsilon$, set $Z_{i}=E_{i-1}$.

If $i \geq N$, go to Step 6).

Else, set $\left(\mathbf{x}^{i}, \boldsymbol{\mu}^{i}\right)=\left(\mathbf{x}^{i-1}, \boldsymbol{\mu}^{i-1}\right), E_{i}=E_{i-1}$ and repeat Step 3$)$.

End.

Else, $Z_{i}=E_{i-1} \cup\left\{\varpi_{i}\right\}$.

End.
Step 4) Solve problem $\operatorname{PDP}\left(Z_{i}\right)$ to obtain a solution $\left(\mathbf{x}^{i}, \boldsymbol{\mu}^{i}\right)$.

Step 5) Choose a set $E_{i} \subset Z_{i}$ with no more than $S+1$ points such that the solution of problem $\operatorname{PDP}\left(E_{i}\right)$ is in the form $\left(\mathrm{x}^{i}, \mu^{i}\right)$. Go to Step 3).

Step 6) Suppose $Z_{i}$ has $k$ points $\varpi_{1}, \varpi_{2}, \ldots, \varpi_{k}$. Starting from $\left(\mathbf{x}^{i}, \boldsymbol{\mu}^{i}, \boldsymbol{\tau}^{i}\right)$, where $\mathbf{x}^{i}$ and $\boldsymbol{\mu}^{i}$ are defined previously and $\tau^{i}=\left[\varpi_{1}, \varpi_{2}, \cdots, \varpi_{k}\right]$ is the $k$ tuple formed by the points in $Z_{i}$, find a local minimum $\left(\mathbf{x}^{*}, \boldsymbol{\mu}^{*}, \boldsymbol{\tau}^{*}\right)$ for problem $\mathbf{P D P} \mathbf{P}_{k}$. Then $\mathbf{x}^{*}$ is taken as the solution for problem $\mathbf{P}$.

In Algorithm 1, we need to initialize two parameters, $N$ and $\epsilon$. The purpose of introducing the integer $N$ is to prevent the algorithm from terminating prematurely. For example, if the iteration number $i$ is small, the subset $\Delta_{i+1}$ may be relatively sparse in $\Delta$ and it may happen that the approximated solution $\mathbf{x}^{i}$, as part of the solution $\left(\mathbf{x}^{i}, \boldsymbol{\mu}^{i}\right)$ of problem $\mathbf{P D P}\left(Z_{i}\right)$, satisfies all the constraints corresponding to index points in $\Delta_{i+1}$. Without introducing the integer $N$, the algorithm would terminate the iteration at this stage even if $g_{\max }\left(\mathbf{x}^{i}, \omega\right)>0$ at some points $\omega \in \Delta \backslash \Delta_{i+1}$ and goes to the final local search procedure. In this case, $\mathrm{x}^{i}$ may not be close enough to the primal solution $\mathbf{x}^{*}$ and the subsequent local search procedure in Step 6) does not find a global solution for problem $\mathbf{P D P} \mathbf{P}_{k}$. The parameter $\epsilon$ relates to the tolerable error. Theoretically, it can be set as close as zero if the Slater condition is satisfied.

The main difference between this algorithm and the conventional one [27] is that this algorithm is more efficient in the sense that the dimension of the quadratic problem in each iteration and that at the final local search procedure is much reduced. In the above algorithm, we can see from Step 5) that the number of points in $E_{i}$ is no more than $S+1$ points. Hence, in Step 3), if $g_{\max }\left(\mathbf{x}^{i-1}, \varpi_{i}\right) \geq \epsilon$, then $Z_{i}=E_{i-1} \cup\left\{\varpi_{i}\right\}$ and the number of points in $Z_{i}$ is no more than $S+2$ points. In Step 3), if $g_{\max }\left(\mathrm{x}^{i-1}, \varpi_{i}\right)<\epsilon$, then $Z_{i}=E_{i-1}$. If $i<N$, then $E_{i}=E_{i-1}$. Hence, the number of points in both $Z_{i}$ and $E_{i}$ is no more than $S+1$ points. As a result, after reiterates of Step 3 ), the number of points in $E_{i}$ and $Z_{i}$ is no more than $S+1$ and $S+2$ points, respectively. According to Algorithm 1, we only need to solve the problem $\operatorname{PDP}(Z)$ in Step 4) and Step 5). As the dimension of the problem $\operatorname{PDP}(Z)$ is equal to the size of the vector $\mathbf{X}$ plus that of $\boldsymbol{\mu}$, the size of the vector $\mathbf{X}$ and $\boldsymbol{\mu}_{j}$ is, respectively, $S \times 1$ and $m \times 1$, and there is no more than $S+2$ points in $Z_{i}$ or there is no more than $S+2 \boldsymbol{\mu}_{j}$, so we only need to solve a quadratic programming problem of dimension not exceeding $S+m(S+2)$ with $S$ linear equality constraints. This is because the number of equality constraints is equal to the size of the vector $\mathbf{Q x}+\mathbf{b}+\sum_{j=1}^{k}\left(\mathbf{A}\left(\omega_{j}\right)\right)^{T} \boldsymbol{\mu}_{j}$, which is $S$. Similarly, we can see that we only need to find a local solution of a nonlinear programming problem at the final local search procedure of dimension no more than $S+(m+1)(S+2)$.

Since the dimension of the optimization problem is greatly reduced, one may query that the numerical accuracy of the obtained solution will be traded off. It is worth noting that if the parameters $\epsilon$ and $N$ are chosen properly, then the efficiency of the improved dual parameterization method will not be traded off with the numerical accuracy of the obtained solution even 
though the dimensionality of the problem is reduced. Or in other words, the solution obtained using the improved dual parameterization method is the same as that of the conventional dual parameterization method. This is because both the conventional and the improved dual parameterization algorithm guarantee that the obtained solution is the globally optimal solution, which is uniquely defined. This result will be proved in Theorem 1 later. In order to prove Theorem 1, we need Lemmas 5 and 6 stated below:

Lemma 5: Step 5) of the Algorithm 1 is numerically feasible.

Proof: Let $Z_{i}=\left\{\omega_{j}: j=1,2, \ldots, k\right\}$. Then problem $\operatorname{PDP}\left(Z_{i}\right)$ is in the form of $\mathbf{P D P}(Z)$. Let $\left(\mathbf{x}_{i}, \boldsymbol{\mu}_{i}\right)$ be the solution to this problem obtained in the $i$ th iteration. Consider the following linear program:

\section{Problem $(\mathbf{L P})_{i}$}

$$
\begin{array}{cc}
\min _{(\boldsymbol{\mu}, \boldsymbol{v})} & \sum_{j=1}^{S+1} v_{j} \\
\text { subject to } & \sum_{j=1}^{k}\left(\mathbf{c}\left(\omega_{j}\right)\right)^{T} \boldsymbol{\mu}_{j}+v_{S+1}=\nu_{i} \\
& \sum_{j=1}^{k}\left(\mathbf{A}\left(\omega_{j}\right)\right)^{T} \boldsymbol{\mu}_{j}+\hat{\boldsymbol{v}}=\tilde{\boldsymbol{c}}_{i} \\
\boldsymbol{\mu} \geq \mathbf{0}
\end{array}
$$

and

$$
v \geq 0
$$

where

$$
\begin{aligned}
\nu_{i} & =\sum_{j=1}^{k}\left(\mathbf{c}\left(\omega_{j}\right)\right)^{T} \boldsymbol{\mu}_{j}^{i} \\
\tilde{\boldsymbol{c}}_{i} & =-\mathbf{Q} \mathbf{x}_{i}-\mathbf{b} \\
\boldsymbol{\mu} & =\left[\begin{array}{lll}
\boldsymbol{\mu}_{1}^{i} & \boldsymbol{\mu}_{2}^{i}, \cdots, & \cdots
\end{array}\right] \\
\hat{\boldsymbol{v}} & =\left[\begin{array}{llll}
v_{1} & v_{2} & \cdots, & v_{S}
\end{array}\right]^{T}
\end{aligned}
$$

and

$$
\boldsymbol{v}=\left[\begin{array}{ll}
\hat{\boldsymbol{v}}^{T} & v_{S+1}
\end{array}\right]^{T} .
$$

In problem $\mathbf{L} \mathbf{P}_{i}$, which is the phase one of a linear program, $v_{j}$ for $j=1,2, \ldots, S+1$ are known as artificial variables in the simplex theory of linear programming. Using the simplex method, we obtain a basic feasible solution $\left(\overline{\boldsymbol{\mu}}_{i}, \overline{\boldsymbol{v}}_{i}\right)$ where $\overline{\boldsymbol{v}}_{\boldsymbol{i}}$ is a zero vector and $\overline{\boldsymbol{\mu}}_{i}$ contains no more than $S+1$ nonzero vectors

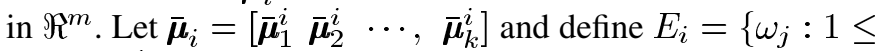
$\left.j \leq k, \boldsymbol{\mu}_{j}^{i} \neq \mathbf{0}\right\}$. We form a new tuple $\hat{\boldsymbol{\mu}}_{i}$ consisting of those column vectors $\overline{\boldsymbol{\mu}}_{j}^{i}$ in $\overline{\boldsymbol{\mu}}_{i}$ whose corresponding indexes $\omega_{j}$ are in $E_{i}$. Then, it is easy to see that $\left(\mathbf{x}_{i}, \hat{\mu}_{i}\right)$ is a solution to problem $\operatorname{PDP}\left(E_{i}\right)$.

This lemma is important for the convergence of the algorithm.

Lemma 6: Let $Z=\left\{\omega_{j}: j=1,2, \ldots, k\right\} \subset \Delta$ be any finite subset of index points. Then problem $\operatorname{PDP}(Z)$ is the Dorn's dual form of the following program:

Problem $(\mathbf{P}(Z))$

$$
\begin{array}{cc}
\min _{\mathbf{x}} & J(\mathbf{x})=\frac{1}{2} \mathbf{x}^{T} \mathbf{Q x}+\mathbf{b}^{T} \mathbf{x}+p \\
\text { subject to } & \mathbf{A}\left(\omega_{j}\right) \mathbf{x}-\mathbf{c}\left(\omega_{j}\right) \leq \mathbf{0}, \text { for } j=1,2, \ldots, k .
\end{array}
$$

A vector $\hat{\boldsymbol{x}} \in \Re^{S}$ is the solution of problem $\mathbf{P}(Z)$ if and only if there exists some $\hat{\boldsymbol{\mu}}=\left[\hat{\boldsymbol{\mu}}_{1} \hat{\boldsymbol{\mu}}_{2} \cdots, \hat{\boldsymbol{\mu}}_{k}\right]$ such that $(\hat{\boldsymbol{x}}, \hat{\boldsymbol{\mu}})$ is a solution of problem $\mathbf{P D P}(Z)$. Furthermore, $V(\mathbf{P}(Z))=$
$-V(\mathbf{P D P}(Z))$, where $V(\mathbf{D})$ denotes the optimal value of a given problem $\mathbf{D}$.

Proof: This lemma is easy to check and we omit the details.

This lemma states the necessary and sufficient relationship between solutions of problem $\mathbf{P}(Z)$ and problem $\mathbf{P D P}(Z)$. Hence, we can use Algorithm 1 to solve the problem $\operatorname{PDP}\left(Z_{i}\right)$ instead. This result is required for Theorem 1 and is stated below.

Theorem 1: If (2) is satisfied, then the sequence $\left\{\mathrm{x}^{i}\right\}$ obtained from Algorithm 1 converges to the solution of problem $\mathbf{P}$. Therefore, assuming that problem $\mathbf{P D P}_{k}$ has only a finite number of local minima for each $k$, if $\epsilon$ and $N$ are suitably chosen, then $\mathbf{x}^{*}$ obtained in Step 6) is the optimal solution of problem $\mathbf{P}$.

Proof: The proof is shown in Appendix I.

The importance of Theorem 1 is that we can guarantee the convergence of Algorithm 1 to the solution of problem $\mathbf{P}$. Since the dimension of the quadratic programming does not exceed $S+m(S+2)$ with $S$ linear equality constraints, Algorithm 1 can be used to solve semi-infinite programming problems efficiently.

\section{NUMERICAL EXPERIMENTS}

We have performed extensive numerical experiments, including the cases with the sets of decimation integers $\{2,4,4\}$, $\{2,6,6,6\}$ and $\{3,3,6,6\}$. We obtain excellent results for all these examples. For brevity and to focus on ideas, we only present the case with the set of decimation integers $\{2,4,4\}$.

Since this set of decimation integers corresponds to a cascade of uniform filter banks in a tree structure manner, an exact perfect reconstruction is possible if the filter lengths are long enough. Hence, there should exist a solution such that the specifications on aliasing and amplitude distortions are strictly satisfied. That means the Slater's constraint qualification holds and our proposed algorithm will converge to the global minimum. Although the existence of a solution or the satisfaction of Slater's constraint is not guaranteed if the filter lengths are short, the possibility of this set of decimation integer getting a solution is higher than the others.

Although perfect reconstruction is easy to achieve if the set of decimation integers is $\{2,4,4\}$ even at short filter lengths, it is not guaranteed that the passband and stopband specifications are satisfied. For real applications, subband processing, such as quantization, is applied. This subband processing is based on the frequency selectivity of the filters. If the frequency selectivity of these filters is bad, then the subband processing may process undesirable frequency bands. As a result, the performance of the overall systems may be bad even though perfect reconstruction is achieved without subband processing. Hence, we sometimes tradeoff the perfect reconstruction conditions with good frequency selectivity filters.

In order to guarantee that the overall system can achieve near perfect reconstruction with the performance better than that reported in [29], in which the amplitude distortion can be bounded by $-40 \mathrm{~dB}$, the aliasing distortion can be bounded by $-60 \mathrm{~dB}$ and the stopband ripple magnitude is bounded by $-60 \mathrm{~dB}$ with 
64 tap filters, we set the specifications on both the real and imaginary parts of amplitude distortions as $-40 \mathrm{~dB}$, and that of the aliasing distortions as well as the specification on the stopband ripple magnitude as $-60 \mathrm{~dB}$.

For this set of decimation integers, the passbands and the stopbands of the filters in the analysis bank are denoted as, respectively, $B_{i}^{p}$ and $B_{i}^{s}$ for $i=0,1,2$, and defined as follows:

$$
\begin{aligned}
B_{0}^{p}= & \left(-\frac{\pi}{2}+\partial B_{0}, \frac{\pi}{2}-\partial B_{0}\right) \\
B_{1}^{p}= & \left(\frac{\pi}{2}+\partial B_{1}, \frac{3 \pi}{4}-\partial B_{1}\right) \\
& \cup\left(-\frac{3 \pi}{4}+\partial B_{1},-\frac{\pi}{2}-\partial B_{1}\right) \\
B_{2}^{p}= & \left(\frac{3 \pi}{4}+\partial B_{2}, \pi\right) \cup\left(-\pi,-\frac{3 \pi}{4}-\partial B_{2}\right) \\
B_{0}^{s}= & \left(\frac{\pi}{2}+\partial B_{0}, \pi\right) \cup\left(-\pi,-\frac{\pi}{2}-\partial B_{0}\right) \\
B_{1}^{s}= & \left(-\pi,-\frac{3 \pi}{4}-\partial B_{1}\right) \\
& \cup\left(-\frac{\pi}{2}+\partial B_{1}, \frac{\pi}{2}-\partial B_{1}\right) \cup\left(\frac{3 \pi}{4}+\partial B_{1}, \pi\right)
\end{aligned}
$$

and

$$
B_{2}^{s}=\left(-\frac{3 \pi}{4}+\partial B_{2}, \frac{3 \pi}{4}-\partial B_{2}\right)
$$

where $\partial B_{0}, \partial B_{1}$ and $\partial B_{2}$ are the transition bandwidths of the lowpass filter, bandpass filter and highpass filter, respectively, and selected as $\partial B_{0}=0.25636, \partial B_{1}=0.3214$, and $\partial B_{2}=$ 0.32099 .

In the improved dual parameterization algorithm, we choose $\epsilon=0.001, N=10$ and $\Delta_{i}$ for $i=1,2, \cdots$ as the set of discrete frequencies sampled from $-\pi$ to $\pi$ with step size 0.01 . The initial values of $\mathbf{x}$ and $\mathbf{y}$ of our algorithm are selected as the filters designed using the Matlab function "fir 1". In fact, any initial condition would give the same globally optimal solution as discussed before.

For the same set of filter lengths employed in [29], our design can achieve the passband ripple magnitudes bounded by $\delta_{p_{0}}=-150.9365 \mathrm{~dB}, \delta_{p_{1}}=-69.1622 \mathrm{~dB}$ and $\delta_{p_{2}}=-98.8590 \mathrm{~dB}$, while the stopband ripple magnitudes bounded by $\delta_{s_{0}}=-72.4579 \mathrm{~dB}, \delta_{s_{1}}=-66.1358 \mathrm{~dB}$ and $\delta_{s_{2}}=-74.1299 \mathrm{~dB}$. Both the real part and imaginary part of the amplitude distortions is less than $-63.1403 \mathrm{~dB}$, and both the real part and imaginary part of the aliasing distortions is less than $-66.6287 \mathrm{~dB}$. It can be checked that our proposed algorithm meet all the required specifications. Fig. 1 shows the responses of the analysis filters and Fig. 2 shows the corresponding amplitude and aliasing distortions. Compared to the result obtained in [29], we have about $20 \mathrm{~dB}$ and 4 $\mathrm{dB}$ improvements on respectively, the amplitude and aliasing distortions, as well as 6-12 $\mathrm{dB}$ improvements on the stopband ripple magnitudes of the filters. The main reasons for the improvements are because the filters obtained in [29] is based on the sum of filter responses of filters in the corresponding
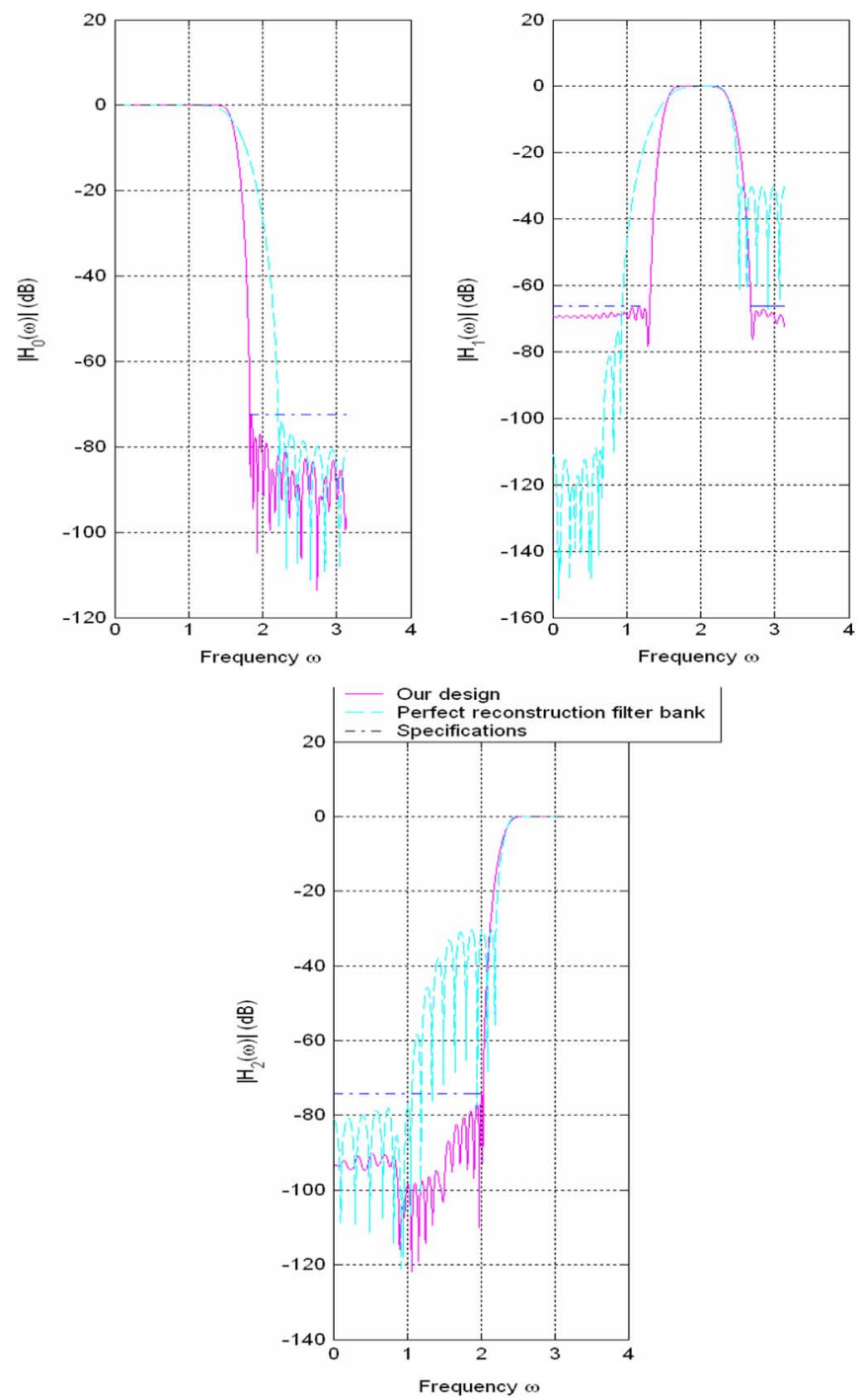

Fig. 1. Magnitude responses of the analysis filters. (Color version available online at http://ieeexplore.iee.org.)

uniform cosine-modulated filter banks, which is not a globally optimal solution in general.

To compare our results with that of perfect reconstruction filter banks, we implement the perfect reconstruction filter banks as a tree structure. There are many filters that the filter lengths of the equivalent filters in the corresponding nonuniform filter banks are less than or equal to 64. For example, we can use a set of filters with filter length 32 in the first branch of the tree structure and 16 in the second branch, or 24 in the first branch and 20 in the second branch, etc. In order to make the roll-off of the frequency response of each filter similar, the filter length of the filters in the first branch should be approximately equal to that in the second branch. Hence, we use 24 tap and 20 tap filters in, respectively, the first branch and second branch. Due to finite bit implementation, the magnitude and aliasing distortions are nonzero and found as, respectively, $-285.8366 \mathrm{~dB}$ and $-279.8754 \mathrm{~dB}$. The passband ripple magnitudes are found as $\delta_{p_{0}}=-31.9776 \mathrm{~dB}, \delta_{p_{1}}=-40.0002 \mathrm{~dB}$ and $\delta_{p_{2}}=-66.3320 \mathrm{~dB}$, while the stopband ripple magnitudes are as $\delta_{s_{0}}=-13.0335 \mathrm{~dB}, \delta_{s_{1}}=-17.2047 \mathrm{~dB}$ 

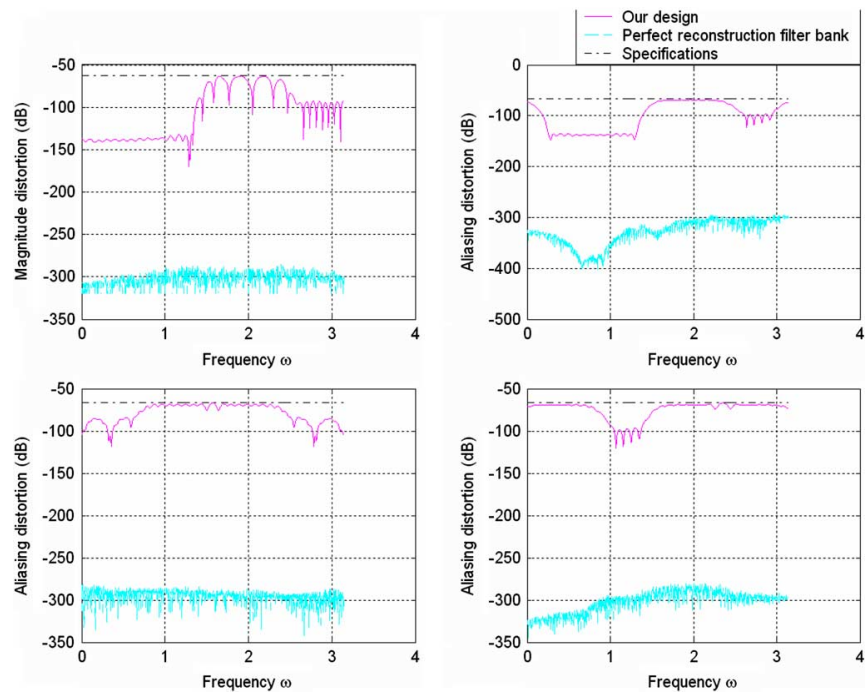

Fig. 2. Amplitude and aliasing distortions. (Color version available online at http://ieeexplore.ieee.org.)

and $\delta_{s_{2}}=-30.2599 \mathrm{~dB}$. This set of filters is far from the required specifications. Compared to our results, we have very significant improvements on both the passband and stopband ripple magnitudes. The main reasons for the improvements are because the filter lengths are too short to satisfy the passband and stopband specifications under the perfect reconstruction condition. So if we relax the perfect reconstruction requirement, better passband and stopband performances can be achieved.

For this example, we see that our algorithm has found a solution satisfying the required constraint specifications. For other nonuniform filter banks with other specifications, in general, there is no guarantee that a solution exists such that the required specifications are satisfied. If there is no solution for a particular set of filter lengths, one may increase the filter lengths incrementally, run the numerical experiments again and check if the required specifications are satisfied. If there is no solution, one may need to relax the specifications.

To compare the computational complexity to the conventional dual parameterization approach [27], we find that both approaches require three iterations for the convergence. However, the conventional approach requires to solve a standard quadratic program of dimension 23472 at the last iteration, while the improved dual parameterization method only requires to solve a standard quadratic program of dimension 6102 at the last iteration. The reduction of the dimensionality of the problem results to the reduction of simulation time not in a linear manner, but in an exponential manner. To understand this point, let's consider the following example. If there are 10 points to search in a one-dimensional problem, then there are 100 points to search in the corresponding two-dimensional problem. Hence, the computational time reduces exponentially with respect to the dimensional of the problem. As a result, the computational complexity of the improved dual parameterization reduces much compared to that of the conventional dual parameterization method. It is found that the simulation time for the improved dual parameterization method is about 5 minutes, while that of the conventional dual parameterization method using the same initial condition and parameters $\epsilon, N$ and $\Delta_{0}$ is about 17 days. All these experiments are running using a PC with Pentium 1.2 GHz CPU and 256 MB DDRAM. Hence, the design time for the improved dual parameterization is greatly reduced by $99.9796 \%$, even though the dimensional of the problem is just reduced by $74.0031 \%$.

\section{CONCLUSION}

The main contribution of this paper is to propose a fast implementation algorithm for solving semi-infinite programming problems and applied to the optimum nonuniform symmetric/antisymmetric linear phase FIR filter bank design problems subject to various practical specifications in the frequency domain. In the improved dual parameterization method, no more than $S+2$ index points are chosen in each iteration of the standard quadratic program to form the parameterized dual problem, where $S$ is the dimension of the primal problem. As a result, it is only required to solve a standard quadratic program of dimension not exceeding $S+m(S+2)$ with $S$ linear equality constraints, where $m$ is the number of inequality constraints of the primal problem. Furthermore, at the final local search procedure, we only need to find a local solution of a nonlinear programming problem of dimension no more than $S+(m+1)(S+2)$. Hence, the improved technique can significantly reduce the computational complexity. The numerical experiments obtained shows a significant improvement in terms of the passband and stopband specifications, as well as the amplitude and aliasing distortions, compared to other methods reported in existing literature.

\section{APPENDIX I \\ PROOF OF THEOREM 1}

According to Lemma 6, we can see that $\mathrm{x}^{i}$ is the solution of the problem $\mathbf{P}\left(Z_{i}\right)$. According to Lemma 5, we see that Step $5)$ is numerically feasible. Hence, according to Step 5), $\mathrm{x}^{i}$ is the solution of problem $\mathbf{P}\left(E_{i}\right)$. On the other hand, $\mathrm{x}^{i+1}$ is the solution of problem $\mathbf{P}\left(Z_{i+1}\right)$ of which the constraint index set $Z_{i+1}$ contains $E_{i}$ as a subset. Thus, it is easy to see that

$$
J\left(\mathrm{x}^{i}\right) \leq J\left(\mathrm{x}^{i+1}\right), \quad \text { for } i=1,2, \cdots
$$

The existence of a Slater point $\mathbf{x}_{0}$ for problem $\mathbf{P}$ shows that the sequence $\left\{J\left(\mathbf{x}^{i}\right)\right\}$ is bounded from above by $J\left(\mathbf{x}_{0}\right)$. Thus, there exists some constant $J^{*}$ such that

$$
J\left(\mathbf{x}^{i}\right) \rightarrow J^{*} \text {, as } i \rightarrow+\infty .
$$

The strict convexity of the quadratic cost $J(\mathbf{x})$ and the boundedness of $\left\{J\left(\mathrm{x}^{i}\right)\right\}$ guarantee that the sequence $\left\{\mathrm{x}^{i}\right\}$ is bounded. Let $\left\{\mathbf{x}_{i_{k}}\right\}$ be any chosen convergent subsequence of $\left\{\mathbf{x}^{i}\right\}$ such that

$$
\mathbf{x}_{i_{k}} \rightarrow \overline{\mathbf{x}} \text {, as } k \rightarrow+\infty
$$

for some $\overline{\mathrm{x}} \in \Re^{S}$. We now show that $\overline{\mathrm{x}}$ is a feasible point of problem $\mathbf{P}$. In fact, if $\overline{\mathbf{x}}$ is not a feasible point of problem $\mathbf{P}$, then there exists $\omega_{0} \in \Delta$ such that $g_{\max }\left(\overline{\mathbf{x}}, \omega_{0}\right)>0$. Let 


$$
\frac{1}{2} g_{\max }\left(\overline{\mathbf{x}}, \omega_{0}\right)=\delta .
$$

Since $g_{\max }(\mathbf{x}, \omega)$ is continuous, we see that there exists $\beta>0$ such that

$$
\begin{aligned}
& \left|g_{\max }(\mathbf{x}, \omega)-g_{\max }\left(\overline{\mathbf{x}}, \omega_{0}\right)\right|<\delta, \\
& \qquad \text { for }\|\mathbf{x}-\overline{\mathbf{x}}\|<\beta \text { and }\left\|\omega-\omega_{0}\right\|<\beta .
\end{aligned}
$$

As a result, we have

$$
g_{\max }(\mathbf{x}, \omega) \geq \delta \text {, for }\|\mathbf{x}-\overline{\mathbf{x}}\|<\beta \text { and }\left\|\omega-\omega_{0}\right\|<\beta .
$$

From (2) and (6), there exists an integer $K$ such that for $k \geq$ $K, \Delta_{i_{k}}$ and $\mathbf{x}^{i_{k}}$ satisfy

$$
\max _{\varpi \in \Delta} \min _{\omega \in \Delta_{i_{k}}}|\varpi-\omega|<\frac{\beta}{2} \text { and }\left\|\mathbf{x}^{i_{k}}-\overline{\mathbf{x}}\right\|<\frac{\beta}{2} .
$$

Especially, there exists $\omega_{i_{k}} \in \Delta_{i_{k}}$ such that $\omega_{i_{k}}$ and $\mathrm{x}^{i_{k}}$ satisfy

$$
\left\|\omega_{i_{k}}-\omega_{0}\right\|<\frac{\beta}{2} \text { and }\left\|\mathbf{x}^{i_{k}}-\overline{\mathbf{x}}\right\|<\frac{\beta}{2} \text { for } k \geq K .
$$

Thus,

$$
g_{\max }\left(\mathbf{x}^{i_{k}}, \omega_{i_{k}}\right) \geq \delta, \text { for } k \geq K \text {. }
$$

It is clear from (12) and the definition of $Z_{i_{k}+1}$ that $\omega_{i_{k}}$ is in $Z_{i_{k}+1}$ and hence

$$
g_{\max }\left(\mathbf{x}^{i_{k}+1}, \omega_{i_{k}}\right) \leq 0, \text { for } k \geq K .
$$

Again, from the definitions of $Z_{i_{k}+1}$ and $E_{i_{k}}$, we see that $\mathbf{x}_{k}^{i}$ and $\mathrm{x}^{i_{k}+1}$ are, respectively, the solution and a feasible point of problem $\mathbf{P}\left(E_{i_{k}}\right)$. Hence, from the fact that the feasible set of problem $\mathbf{P}\left(E_{i_{k}}\right)$ is convex and its objective function is strictly convex, $J(\mathbf{x})$ is strictly monotone along the segment connecting $\mathbf{x}_{k}^{i}$ and $\mathbf{x}^{i_{k}+1}$. Particularly, we have

$$
J\left(\mathbf{x}^{i_{k}}\right)<J\left(\frac{\mathbf{x}^{i_{k}}+\mathbf{x}^{i_{k}+1}}{2}\right)<J\left(\mathbf{x}^{i_{k}+1}\right) .
$$

Since $\left\{\omega_{i_{k}}\right\}$ is contained in the compact set $\Delta$, it has a converging subsequence. Without loss of generality, we suppose $\left\{\omega_{i_{k}}\right\}$ itself converge to $\omega^{\prime} \in \Delta$. At the same time, we can further suppose that $\left\{\mathbf{x}^{i_{k}+1}\right\}$ converges to some limit $\hat{\mathbf{x}}$. Taking limit $(k \rightarrow+\infty)$ in (13) and (14), we obtain

$$
g_{\max }\left(\hat{\mathbf{x}}, \omega^{\prime}\right) \leq 0
$$

and

$$
J(\overline{\mathbf{x}}) \leq J\left(\frac{\overline{\mathbf{x}}+\hat{\mathbf{x}}}{2}\right) \leq J(\hat{\mathbf{x}}) .
$$

From (15), it allows that

$$
\begin{aligned}
\left|g_{\max }\left(\hat{\mathbf{x}}, \omega^{\prime}\right)-g_{\max }\left(\overline{\mathbf{x}}, \omega_{0}\right)\right| & \\
& \geq g_{\max }\left(\overline{\mathbf{x}}, \omega_{0}\right)-g_{\max }\left(\hat{\mathbf{x}}, \omega^{\prime}\right) \geq 2 \delta .
\end{aligned}
$$

According to (11), we have $\left\|\omega^{\prime}-\bar{\omega}\right\| \leq \beta / 2$. Hence, (8) shows that

$$
\|\hat{\mathbf{x}}-\overline{\mathbf{x}}\| \geq \beta
$$

From (5), we have

$$
J(\overline{\mathbf{x}})=J(\hat{\mathbf{x}}) .
$$

Now we see that (16), (18), and (19) contradict the fact that $J(\mathbf{x})$ is strictly convex. Therefore, $\overline{\mathbf{x}}$ is feasible to problem $\mathbf{P}$.

Next we show that the whole sequence $\left\{\mathbf{x}^{i}\right\}$ converges to the solution $\mathbf{x}^{*}$ of problem $\mathbf{P}$. Suppose $\left\{\mathbf{x}^{i}\right\}$ does not converge. Then there are two sequences $\left\{\mathbf{x}^{i_{k}}\right\}$ and $\left\{\mathbf{x}^{j_{k}}\right\}$ converging to $\mathrm{x}^{\prime}$ and $\mathrm{x}^{\prime \prime}$, respectively, where $\mathrm{x}^{\prime} \neq \mathrm{x}^{\prime \prime}$. From the above, we can see that both $\mathbf{x}^{\prime}$ and $\mathbf{x}^{\prime \prime}$ are feasible to problem $\mathbf{P}$. Then point $\left(\mathbf{x}^{\prime}+\mathbf{x}^{\prime \prime}\right) / 2$ is feasible to problem $\mathbf{P}$ and hence feasible to problem $\mathbf{P}\left(Z_{i_{k}}\right)$ for all $k \geq 1$. Therefore

$$
J\left(\frac{\mathrm{x}^{\prime}+\mathrm{x}^{\prime \prime}}{2}\right)<\frac{J\left(\mathbf{x}^{\prime}\right)+J\left(\mathbf{x}^{\prime \prime}\right)}{2}=J^{*} .
$$

Since $J\left(\mathbf{x}_{k}^{i}\right) \rightarrow J^{*}$ as $k \rightarrow+\infty$, we have

$$
J\left(\frac{\mathbf{x}^{\prime}+\mathbf{x}^{\prime \prime}}{2}\right)<J\left(\mathbf{x}^{i_{k}}\right)
$$

for sufficiently large $k$. This contradicts to the fact that $\mathbf{x}^{i_{k}}$ is the solution to problem $\mathbf{P}\left(Z_{i_{k}}\right)$. Therefore, $\left\{\mathbf{x}^{i}\right\}$ converges to $\mathbf{x}^{*}$. It is clear that $\mathbf{x}^{*}$ is the solution of problem $\mathbf{P}$.

Finally, if $\epsilon$ is sufficiently small and if $N$ is sufficiently large, then the approximation solution $\mathrm{x}^{i}$ found at the termination of the iteration in Step 5) will be so close to the primal solution that the objective value of problem $\mathbf{P D P}_{k}$ at $\left(\mathbf{x}^{i}, \boldsymbol{\mu}^{i}, \boldsymbol{\tau}^{i}\right)$ is smaller than the second smallest local minimum value of problem $\mathbf{P D P}_{k}$. Therefore, the final local search procedure will find the global solution. And this completes the proof.

\section{REFERENCES}

[1] J. D. Gorman and A. O. Hero, "Lower bounds for parametric estimation with constraints," IEEE Trans. Inf. Theory, vol. 26, no. 6, pp. 1285-1301, 1990.

[2] A. Garulli, A. Vicino, and G. Zappa, "Conditional central algorithms for worst case set-membership identification and filtering," IEEE Trans. Autom. Control, vol. 45, no. 1, pp. 14-23, 2000.

[3] S. Nordebo, Z. Zang, and I. Claesson, "A semi-infinite quadratic programming algorithm with applications to array pattern synthesis," IEEE Trans. Circuits Syst. II, Analog Digit. Signal Process., vol. 48, no. 3, pp. 225-232, 2001.

[4] S. Nordebo, I. Claesson, and Z. Zang, "Optimum window design by semi-infinite quadratic programming," IEEE Signal Process. Lett., vol. 46, no. 10, pp. 262-265, 1999.

[5] H. H. Dam, K. L. Teo, S. Nordebo, and A. Cantoni, "The dual parameterization approach to optimal least square FIR filter design subject to maximum error constraints," IEEE Trans. Signal Process., vol. 48, no. 8, pp. 2314-2320, 2000 
[6] S. Nordebo and Z. Zang, "Semi-infinite linear programming: A unified approach to digital filter design with time- and frequency-domain specifications," IEEE Trans. Circuits Syst. II, Analog Digit. Signal Process., vol. 46, no. 9, pp. 765-775, 1999.

[7] R. Vuerinckx, "Design of high-order Chebyshev FIR filters in the complex domain under magnitude constraints," IEEE Trans. Signal Process., vol. 46, no. 6, pp. 1676-1681, 1998.

[8] Z. Zhu, H. Leung, and S. Wang, "Design of complex FIR filters using semi-infinite quadratic optimization techniques," Electron. Lett., vol. 35 , no. 4, pp. 270-271, 1999.

[9] H. H. Dam, S. Nordebo, A. Cantoni, and K. L. Teo, "Frequency domain design for digital Laguerre networks," IEEE Trans. Circuits Syst. I, Fundam. Theory Appl., vol. 47, no. 4, pp. 578-581, 2000.

[10] Z. Zhu, S. Wang, H. Leung, and Z. Ding, "Matrix filter design using semi-infinite programming with application to DOA estimation," IEEE Trans. Signal Process., vol. 48, no. 1, pp. 267-271, Jan. 2000.

[11] B.-N. Vo, A. Cantoni, and K. L. Teo, "Envelope constrained filter with linear interpolator," IEEE Trans. Signal Process., vol. 45, no. 6, pp. 1405-1414, Jun. 1997.

[12] C. H. Tseng, K. L. Teo, Z. Zang, and A. Cantoni, "A dual approach to continuous-time envelope-constrained filter design via orthonormal filters," IEEE Trans. Circuits Systems I, Fundam. Theory Appl., vol. 46, no. 9, pp. 1042-1054, 1999.

[13] C. H. Tseng, K. L. Teo, A. Cantoni, and Z. Zang, "Design of robust envelope-constrained filter with orthonormal bases," IEEE Trans. Signal Process., vol. 48, no. 10, pp. 2881-2891, Oct. 2000.

[14] R. G. Shenoy, D. Burnside, and T. W. Parks, "Linear periodic systems and multirate filter design," IEEE Trans. Signal Process., vol. 42, no. 9, pp. 2242-2256, Sep. 1994.

[15] P. Moulin, M. Anitescu, K. O. Kortanek, and F. A. Potra, "The role of linear semi-infinite programming in signal-adapted QMF bank design," IEEE Trans. Signal Process., vol. 45, no. 9, pp. 2160-2174, Sep. 1997.

[16] P. Moulin and M. K. Mihçak, "Theory and design of signal-adapted FIR paraunitary filter banks," IEEE Trans. Signal Process., vol. 46, no. 4, pp. 920-929, Apr. 1998.

[17] C. Y.-F. Ho, B. W.-K. Ling, Y.-Q. Liu, P. K.-S. Tam, and K.-L. Teo, "Design of nonuniform near allpass complementary FIR filters via a semi-infinite programming technique," IEEE Trans. Signal Process., vol. 53, no. 1, pp. 376-380, Jan. 2005.

[18] — - "Optimal design of nonuniform FIR transmultiplexer using semiinfinite programming," IEEE Trans. Signal Process., vol. 53, no. 7, pp. 2598-2603, Jul. 2005

[19] M. A. Coffey and D. M. Etter, "Multiresolution analysis on bounded domains for the design of biorthogonal wavelet bases," IEEE Trans. Signal Process., vol. 50, no. 3, pp. 509-519, Mar. 2002.

[20] K. Shimizu, S. Fujimaki, and Y. Ishizuka, "Constrained optimization methods in Hilbert spaces and their applications to optimal control problems with functional inequality constraints," IEEE Trans. Autom. Control, vol. AC-31, no. 6, pp. 559-564, 1986.

[21] E. Polak and D. M. Stimler, "Majorization: A computational complexity reduction technique in control system design," IEEE Trans. Autom. Control, vol. AC-33, no. 11, pp. 1010-1021, 1988.

[22] M. A. Dahleh and J. Boyd Pearson, JR., "Optimal rejection of persistent disturbances, robust stability, and mixed sensitivity minimization," IEEE Trans. Autom. Control, vol. AC-33, no. 8, pp. 722-731, 1988.

[23] M. E. Halpern, "Rational suboptimal continuous-time controller design," IEEE Trans. Autom. Control, vol. 45, no. 9, pp. 1731-1734, 2000.

[24] J. L. Figueroa and J. A. Romagnoli, "An algorithm for robust pole assignment via polynomial approach," IEEE Trans. Autom. Control, vol. 39 , no. 4, pp. 831-835, 1994.

[25] K. L. Teo and C. J. Goh, "A simple computational procedure for optimization problems with functional inequality constraints," IEEE Trans. Autom. Control, AC-32, no. 10, pp. 940-941, 1987.

[26] H. Mine, M. Fukushima, and Y. Tanaka, "On the use of $\epsilon$-most-active constraints in an exact penalty function method for nonlinear optimization," IEEE Trans. Autom. Control, AC-29, no. 11, pp. 1040-1042, 1984.

[27] S. Ito, Y. Liu, and K. L. Teo, "A dual parameterization method for convex semi-infinite programming," Ann. Oper. Res., vol. 98, pp. 189-213, 2000.

[28] M. S. Bazarra and C. M. Shetty, Nonlinear Programming: Theory and Algorithm. New York: Wiley, 1979.

[29] J. Li, T. Q. Nguyen, and S. Tantaratanna, "A simple design method for near-perfect-reconstruction nonuniform filter banks," IEEE Trans. Signal Process., vol. 45, no. 8, pp. 2105-2109, Aug. 1997.

[30] S.-Y. Wu and S. C. Fang, "Solving convex programs with infinitely many linear constraints by a relaxed cutting plane method," Comput. Math. Appl., vol. 38, pp. 23-33, 1999.

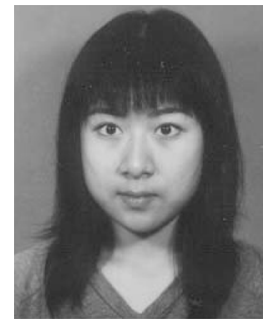

Charlotte Yuk-Fan Ho received the B.Eng. (Hons.) degree in Electrical and Electronic Engineering from the Hong Kong University of Science and Technology, Hong Kong, China, in 2000 and the M.Phil. degree in Electronic and Information Engineering, Hong Kong Polytechnic University, Hong Kong, China, in 2003. She is currently working towards the $\mathrm{Ph}$.D. degree in mathematical sciences and electronic engineering at Queen Mary, University of London, London, U.K.

Her research interests include solving optimization problems having infinite constraints with applications to filters, filter banks, and sigma-delta modulators designs; analyzing systems having discontinuous nonlinearity with applications to sigma-delta modulators and digital filters with two's complement arithmetic; studying filter banks and wavelets theory with applications to multimedia and biomedical signal processing; and investigating fuzzy, impulsive, and optimal control theory with applications to sigma-delta modulators and power electronic systems. She has published eight IEEE transactions papers, five other international journal papers, and nine international conference papers in the above areas.

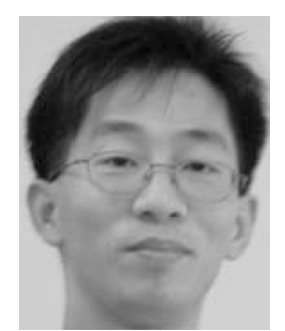

Bingo Wing-Kuen Ling received the B.Eng. (Hons.) and M.Phil. degrees in electrical and electronic engineering from the Hong Kong University of Science and Technology, Hong Kong, China, in 1997 and 2000 , respectively, and the Ph.D. degree in electronic and information engineering from the Hong Kong Polytechnic University, Hong Kong, China, in 2003.

In 2004, he joined the King's College London, London, U.K., as a Lecturer. His research interests include solving optimization problems having infinite constraints with applications to filters, filter banks, and sigma-delta modulators designs; analyzing systems having discontinuous nonlinearity with applications to sigma-delta modulators and digital filters with two's complement arithmetic; studying filter banks and wavelets theory with applications to multimedia and biomedical signal processing; and investigating fuzzy, impulsive, and optimal control theory with applications to sigma-delta modulators and power electronic systems.

Dr. Ling served as a reviewer of several IEEE and IEE journals, and as a reviewer and member of technical committees of international conferences as well.

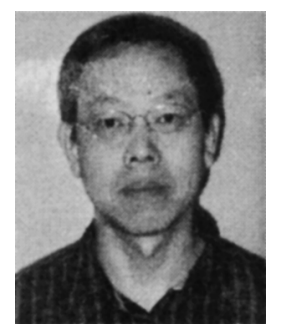

Yan-Qun Liu received the Ph.D. degree in mathematics from the University of Western Australia, Australia, in 1997.

From 1997 to 1999, he was a Research Associate with the School of Mathematics and Statistics, Curtin University of Technology, Perth, VVA, Australia. From 2000 to 2003, he was a Research Fellow in the Department of Applied Mathematics and, from 2003 to 2004, a Senior Research Fellow in the Department of Electrical and Information Engineering, both at the Hong Kong Polytechnic University, Hong Kong, China. Currently, he is a Lecturer in the Department of Mathematics and Statistics, Royal Melbourne Institute of Technology (RMIT) University, Melbourne, Australia. His research interests include optimization, optimal control, and signal processing.

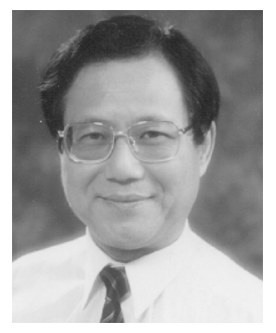

Peter Kwong-Shun Tam (S'74-M'76) received the B.E., M.E., and Ph.D. degrees from the University of Newcastle, Newcastle, Australia, in 1971, 1973, and 1976, respectively, all in electrical engineering.

From 1967 to 1980 , he held a number of industrial and academic positions in Australia. In 1980, he joined the Hong Kong Polytechnic University, Kowloon, Hong Kong, China, as a Senior Lecturer, where he was an Associate Professor in the Department of Electronic and Information Engineering. His research interests include signal processing, automatic control, fuzzy systems, and neural networks.

Dr. Tam has participated in the organization of a number of symposiums and conferences. 


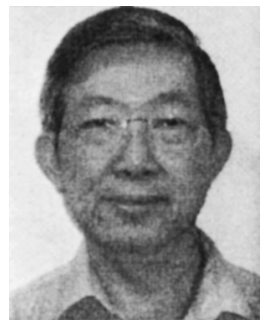

Kok-Lay Teo (M'74-SM'87) was born in Johore, Malaysia, in 1946. He received the B.Sc. degree in telecommunications engineering from Ngee Ann Technical College, Singapore, in 1969 and the M.A.Sc. and Ph.D. degrees in electrical engineering from the University of Ottawa, Ottawa, ON, Canada, in 1971 and 1974 , respectively.

From 1974 to 1985, he was with the Department of Applied Mathematics, University of New South Wales, Australia, and from 1985 to 1987, he was with the Department of Industrial and Systems Engineering, National University of Singapore, Singapore. In 1988, he returned to Australia as an Associate Professor in the Department of Mathematics, University of Western Australia, Australia. In 1996, he joined the Department of Mathematics and Statistics, Curtin University of Technology, Perth, WA, Australia, as a Professor. From 1999 to 2004, he was Chair Professor of Applied Mathematics and Head of Department of Applied Mathematics at the Hong Kong Polytechnic University, Hong Kong, China. Currently, he is Professor of Applied Mathematics and Head of Department of Mathematics and Statistics at Curtin University of Technology. He has delivered several keynote and fully funded invited lectures and published five books, over 250 journal papers, and a number of conference papers. The software package MISER3.3, used for solving general constrained optimal control problems, was developed by the research team under his leadership. His research interests include both the theoretical and practical aspects of optimal control and optimization and their practical applications, such as in signal processing in telecommunications and financial portfolio optimization

Dr. Teo is Editor-in-Chief of the Journal of Industrial and Management Optimization. He also serves as an Associate Editor of a number of international journals, including Automatica; Nonlinear Dynamics and Systems Theory; Journal of Global Optimization Engineering and Optimization; Discrete and Continuous Dynamic Systems (Series A and Series B); and Dynamics of Continuous, Discrete and Impulsive Systems (Series A and Series B). He has also edited special issues for several journals, including Annals of Operations Research; Journal of Global Optimization; and Dynamics of Continuous, Discrete and Impulsive Systems (both Series A and Series B). 